\title{
Australian hepatic types at the National Herbarium of New South Wales (NSW) and the Ray Herbarium (SYD)
}

\author{
E.A. Brown, H.P. Ramsay and J. Seur
}

\begin{abstract}
E.A. Brown, Ramsay, H.P. E Seur, J. (National Herbarium of New South Wales, Royal Botanic Gardens, Sydney, NSW, Australia 2000) 1992. Australian hepatic types at the National Herbarium of New South Wales (NSW) and the Ray Herbarium (SYD). Telopea 5(1): 67-90. The National Herbarium of New South Wales and the Ray Herbarium in Sydney, New South Wales hold important collections of type specimens of Austalian hepatics. A total of 99 names are listed here, together with details of the protologue, complete label data for each specimen, other herbaria known to hold part of the type, and recent information concerning the status of the name. Included are many Stephani and Carrington and Pearson names as well as more recent ones described by Hewson and Na-Thalang.
\end{abstract}

\section{Introduction}

While the Hepatic Collections in the two herbaria in Sydney, New South Wales, are not large [6000 specimens, 100 types (NSW) and 2284 specimens, $<10$ types (SYD)] (Ramsay \& Seur 1990), type specimens of many Australian and exotic species are present. Particularly significant collections held at the National Herbarium of New South Wales [NSW] are those of T. Whitelegge, W.W. Watts (including some from W. Gunn), and H.J. Hewson; and at the Ray Herbarium, University of Sydney [SYD], those of Hewson and $\mathrm{O}$. Na-Thalang. The main geographical regions represented by the types are Australia including Lord Howe Island (Whitelegge, Watts, Na-Thalang), presented in this paper, and Vanuatu [New Hebrides] (W. Gunn) and Papua New Guinea (Hewson) to be reported separately.

The earliest descriptions of New South Wales hepatics are those of B. Carrington \& W.H. Pearson (1888) who determined and published a list of Whitelegge's collections. R.H. Bastow's work on Tasmanian hepatics $(1888,1914)$ also included reference to many New South Wales collections. Watts published several papers on Australian hepatics (1902, 1903, 1905) and left a manuscript (1912), as part of a Census of New South Wales Plants, that he had intended publishing in the same way as he had done for the mosses (Watts \& Whitelegge 1902, 1906). In this unpublished manuscript he listed species, localities and collector's names for 205 Hepatics and 10 Anthocerotes. The names contained in this unpublished manuscript include many described by F. Stephani based on specimens sent to him for identification (Stephani 1884, 1889; Stephani \& Watts 1914).

The cryptogamic collection at NSW was increased greatly in the late 19th and early 20th century by W.W. Watts. After his departure to Victoria in 1916 (Ramsay 1980) curation and addition to the collections was very limited. Conditions at NSW were extremely cramped until 1982 when a new building opened and the W.W. Watts room was set aside for cryptogams.

A period of inactivity in collecting and describing hepatics in New South Wales persisted from 1920 until the mid 1960s, when taxonomic research was undertaken 
by G.K. Berrie and R. Carolin's students Hewson and Na-Thalang, at Sydney University. In 1963 Berrie published Haplomitrium intermedium, the first member of the Calobryales recorded in Australia, from a type collected in the Blue Mountains (Berrie 1963). Hewson $(1970 a, b)$ revised the family Aneuraceae in Australia and New Guinea while Na-Thalang (1980) revised the genus Riccia in Australia. Hewson and $\mathrm{Na}$-Thalang types are located either at SYD or NSW and in some cases at both.

There is still much to be learnt about hepatics in New South Wales; we do not even have a complete species list for this State in which both subtropical and temperate taxa occur. Some recent publications (Ramsay et al. 1990, Downing et al. 1991) and further studies being undertaken by H.P. Ramsay et al. list the hepatics for specific areas in N.S.W. and form the basis for the eventual production of a Census of New South Wales hepatics.

Locating types is essential for taxonomic revisions and a register providing details and locations of type specimens can be useful. Ramsay \& Seur (1990) and Ramsay et al. (1990) outlined the methods for collection and recording of data for a register of type collections of mosses. Their investigations highlighted the often incomplete information in many type citations in taxonomic literature (e.g. date of collection and collector's number). Since most descriptions of species of hepatics published prior to the 1970s do not cite the herbarium holding the type the holotype cannot be clearly defined. Examining the type specimen and its label data may give important details not included in the protologue.

\section{Method}

In this paper we deal only with those hepatic type specimens of Australian species held in the two herbaria named NSW and SYD.

The studies were carried out in a series of steps:

1. All data on each herbarium packet containing specimens designated as types or $\mathrm{sp}$. nov. (i.e. putative types) were recorded on index cards.

2. The type description for each taxon was obtained and the label data compared with that in the protologue. Where these matched, the specimen is recorded as a probable type.

3. Where possible, reference is made to the herbarium holding the major collections of the author of the basionym if this is not NSW or SYD. In most cases, except for species named after the mid 1970s, the holotypes are held in European herbaria and specimens in Australia usually are isotypes. For a number of species a series of specimen numbers were listed with the type description and are syntypes and it may be desirable, therefore, when taxonomic revisions are carried out, to designate a lectotype at that time.

4. References are given for recent studies, name changes, lectotypification etc. for each taxon.

5. To ensure that we have detected all relevant type specimens held at NSW and SYD, we checked publications and searched through all collections at NSW and SYD for specimens not labelled as types but carrying the original label data. As the status of quite a number of specimens had been overlooked in the repackaging at NSW during the early 1980s these further checks, although time-consuming, proved rewarding.

6. The information is now stored on disk and will remain available for future updating. 
The register of type specimens of Australian hepatics presented here has been arranged alphabetically by genera and species based on the name for which the specimen is a type. The list contains the original name for each taxon and reference, the protologue [Proto] (collection details as cited in the protologue), full label data [Label] for each separate specimen (label 1,2 etc.) at NSW and/or SYD if more than one was present. Also listed are location and status of all types [Types] where known (from examination or from literature), synonymy where applicable, as well as any other recent information such as lectotypification [Comment].

At some time in the past many of Watts' collections have been broken up, repacketted and relabelled; the duplicates may all be contained within the one specimen folder or sometimes they have been separated. Where label information does not differ significantly between packets it is usually listed only once, with a note that there are 2 or more specimens; sometimes information on packets within the same specimen folder differs markedly and this has been recorded. The labels on the outer specimen folders has not been recorded as it is only a more recent copy of the information on the Watts' packets.

No taxonomic decisions other than those already in the literature are presented here, nor is lectotypification of any species implied. This is particularly important for taxa described by Stephani; in his later years he broke up many of his specimens and sold duplicates to BM, FH and other institutions. Some of these duplicates, when they come from mixed collections, are not the taxon referred to in the protologue and great care must be taken when typifying. It is probably unwise and inaccurate, therefore, to cite holotypes and this has not been done, even when Stephani specified a collector's number. Herbaria, other than NSW and SYD, holding types have been listed from Index Hepaticarum. Index Hepaticarum VIII (Bonner 1976) and prior volumes were used to obtain putative label information for many of the types held at $G$. E.A. Brown has examined the type specimens from overseas herbaria for the genera Riccardia and Aneura in the course of other studies (Brown \& Braggins 1989).

\section{Register of names}

Aneura aequicellularis Stephani (Stephani \& Watts 1914: 95)

РRото: 'Australia, New South Wales (Wentworth Falls): Watts legit No. 117 [1117].'

Label: New South Wales: Wentworth Falls, cave, W.W. W[atts] 1117, Sep 1912.

TYPES: G (lectotype), NSW (isolectotype).

Comment: = Riccardia aequicellularis (Stephani) Hewson fide Hewson (1970b: 79). Lectotypified by Brown \& Braggins (1989: 26).

Aneura athertonensis Hewson (1970a: 188)

Pвото: 'Holotype - Charmillan Creek, Tully Falls Road, Atherton Tableland, on basalt rock on creek bank in disturbed rain-forest, Qld. Hewson, 422, 8.1964 (NSW): Isotype (BRI).'

LABEL: QUEENSLAND: Charmillan Creek, rock, rainforest, basalt, H.J. Hewson H.64.71.422, 22 Aug 1964.

TYPES: NSW (179239: holotype), BRI (isotype).

Comment: Name in current use. 
Aneura coriacea Stephani (1917: 23)

PRoto: 'Australia: New South Wales (Watts legit.).'

Label: New South Wales: Katoomba Falls, W.W. Watts 567, 15 Jan 1902.

TYPES: G (11036: lectotype), NSW (H241: isolectotype).

COMMENT: No particular specimen cited in protologue. The NSW specimen has been annotated by Hewson: 'Isotype of $A$. coriacea = Riccardia crassa, published Hewson $1970 b^{\prime}$. Lectotypified by Brown \& Braggins (1989: 85). Specimen in G seen by Stephani is Watts 567 (Icones Hep. 474).

\section{Aneura eachamensis Hewson (1970a: 184)}

PRoTo: 'Holotype - Vision Falls, Lake Eacham, Atherton Tableland, on basalt rock in rain-forest, Queensland. Hewson, 398, 8.1964 (NSW): Isotype (BRI).'

LABEL: QueENSLAND: Vision Falls, Lake Eacham, H.J. Hewson H.64.68.398, 20 Aug 1964. TYPES: NSW (179240: holotype), BRI (isotype).

COMMENT: Name in current use.

Aneura gigantea Stephani (Stephani \& Watts 1914: 95)

PRoto: 'Australia, N.S. Wales, (Cambewarra): Watts legit No. 920.'

LABEL: New SOUTH WALES: under cliff, above Baldy's, Cambewarra,

W.W. Watts 920, 18 Oct 1907 [3 packets in one specimen folder].

TYPES: G (11042), NSW.

CoMment: = Aneura alterniloba (Hook. f. \& Taylor) Taylor \& Hook. f. in Gottsche, Lindenb. \& Nees var. gigantea (Stephani) Hewson; G 11042 and '920 NSW' are cited as the holotype and isotype respectively by Hewson (1970a: 187).

Aneura pusilla Stephani (Stephani \& Watts 1914: 96)

Proto: 'Australia, (Old Railway Cutting, Blackheath): Watts legit, 1051.'

LABEL: NeW SOUTH WALES: old railway cutting, Blackheath, W.W. Watts 1051, Jan 1911 [3 packets in one specimen folder].

TYPES: G (11049: lectotype), NSW (H242: isolectotype).

COMment: Listed as a synonym of Riccardia crassa (Schwaegr.) Carrington \& Pearson (Hewson 1970a: 104). Taxon reinstated and transferred to Riccardia; lectotypified by Brown \& Braggins (1989: 82).

Aneura rodwayi Hewson (1970a: 188)

PRoto: 'Holotype-Hartz River, Mt. Hartz National Park, on clay soil near river bank, Tas., Hewson, 215, 9.1963, (NSW.).'

LABEL: TASMANIA: Hartz River, Mt Hartz, on soil near river, H.J. Hewson H63.33.215, 10 Sep 1963.

TYPE: NSW (179241: holotype).

COMMENT: Name in current use.

Aneura rufescens Stephani (Stephani \& Watts 1914: 96)

Proto: 'Australia, New South Wales, (National Pass, Wentworth Falls): Watts legit, 1124.' 
Label: New South Wales: National Pass, Wentworth Falls, W.W. Watts 1124, Sep 1912 [2 packets in one specimen folder].

TYPES: G (11050: lectotype), NSW (H243: isolectotype).

Comment: = Riccardia crassa fide Hewson (1970b: 104). Lectotypified by Brown \& Braggins (1989: 85).

\section{Aneura rupicola Stephani (1917: 41)}

PRoto: 'Australia: New South Wales (Watts legit.).'

Label: New South Wales: 1. Shaws Bay, log, W.W. Watts 579, 20 Sep 1901; 2. Shaws Bay, Ballina, R[ichmond] R[iver], log, W.W. W[atts] 579, 20 Sep 1901 [both packets are in one specimen folder].

TYPES: G (11051 ex Hb Levier 3388), NSW (H248).

COMmeNT: G specimen annotated 'Original. Shaw's Bay, east Ballina. Icones Hep. 520'. = Riccardia rupicola (Stephani) Hewson; G 11051 cited as holotype and 'NSW 579' as isotype by Hewson (1970b: 99).

Aneura walesiana Stephani (Stephani \& Watts 1914: 97)

Proto: 'Australia, New South Wales, (Blackheath): Watts legit, 1023.'

LABEL: New SOUTH WalEs: Horseshoe Falls, Blackheath, wet cliff, W.W. W[atts] 1023, 7 Jan 1911 [3 packets in one specimen folder].

TYPES: G (lectotype), NSW (H244: isolectotype).

COMMENT: = Riccardia crassa fide Hewson (1970b: 104). Lectotypified by Brown \& Braggins (1989: 85).

\section{Aneura wattsiana Stephani (1917: 46)}

PROTo: 'Australia, New South Wales (Watts legit.).'

Label: New South Wales: 1. E Ballina, [Richmond River], on tree fern, wet places, W.W. W[atts] 237, 2 Mar 1901 (NSW H252); 2. label identical, no NSW number.

TYPES: G (11057 ex Hb Levier 3018), NSW.

COMMENT: No specific locality or specimen number cited in protologue. Specimen in G (11057) has the following data 'W.W. Watts 237, 1901, Ballina, New South Wales. Icones Hep. 299'. = Riccardia wattsiana (Stephani) Hewson; G 11057 cited as holotype and 'NSW 237' as isotype by Hewson (1970b: 91). The un-numbered NSW specimen has a second Watts collection attached to it (Watts 346).

Archilejeunea australis Stephani. (1911: 734)

Proto: 'Australia, New South Wales.'

LABEL: NeW SOUTH WALES: back of Wickham's, nr. Ballina, tree, W.W. Watts 415, 3 June 1902.

TYPES: G (ex Hb Levier 3483), NSW.

COMMENT: = Thysananthus australis (Stephani) Thiers \& Gradstein; specimen in G (ex $\mathrm{Hb}$ Levier 3483-Watts 415) cited as the holotype by Thiers \& Gradstein (1989: 66); no reference is made to the $G$ specimen being labelled as the type by Stephani. 
Balantiopsis decurrens Stephani (Stephani \& Watts 1914: 98)

Proto: 'Australia, (Wyong): Watts, 985.'

LABEL: NeW SOUth Wales: gully south of house, 'Kingwell', Wyong, W.W. Watts 985, Nov 1907 [3 packets in one specimen folder].

TYPES: G, NSW.

COMMENT: = B. diplophylla (Hook. f. \& Taylor) Mitten fide Engel (1968: 109, 111) .

Balantiopsis hastatistipula Stephani (Stephani \& Watts 1914: 98)

Proto: 'Australia, (Blackheath), Watts, 1052.'

LABEL: New SOUTH Wales: Blackheath, old railway cutting, W.W. Watts 1052, Jan 1911 [2 packets in one specimen folder].

TYPES: G, NSW.

COMMENT: = Isotachis sp. fide Engel (1968: 118).

Balantiopsis kingwella Stephani (Stephani \& Watts 1914: 99)

Proto: 'Australia (Kingwell, Wyong): Watts 943.'

Label: New South Wales: gully south of house, 'Kingwell', Wyong, W.W. Watts 943, Nov 1907 [3 packets in one specimen folder].

TYPES: G, NSW.

COMMENT: $=$ B. diplophylla fide Engel (1968: 109, 111).

Balantiopsis subkingwella Stephani (Stephani \& Watts 1914: 99)

Proto: 'Australia (Kingwell, Wyong): Watts 945, 971 a.

Label: New South Wales: 1. 'Kingwell', Wyong, W.W. W[atts] 945, 20 Aug 1909; 2. 'Kingwell', Wyong, gully south of house, W.W. Watts 971a, Nov 1907 (NSW H285) [2 packets in one specimen folder].

TYPES: G, NSW.

COMMENT: $=$ B. diplophylla fide Engel $(1968: 109,111)$. Watts $971 b$ is the type of Mastiogobryum corbieri Stephani, see below.

Brachiolejeunea robusta Stephani (1912: 141)

PROTO: 'Australia. New South Wales.'

Label: New South Wales: 1. Hopkins Creek, Stanwell Park, rocks, W.W. W[atts] 830, May 1905; 2. Hopkins Creek, Stanwell Park, [W.W.] Watts 830, May 1905.

TYPES: FH, G (ex Hb Levier 4882), NSW, U.

COMmENT: Specimen in G (Hb Levier 4882-Watts 830) cited as holotype and $\mathrm{FH}$ as isotype by Thiers \& Gradstein (1989: 64) but there is no supporting discussion. Reduced to synonymy under Spruceanthus thozetianus (Gottsche \& F. Muell.) Thiers \& Gradstein.

Chiloscyphus maximus Stephani (Stephani \& Watts 1914: 103)

Proto: 'Australia (Etta's Glen, Black Spur, Vict.): Watts, 968.'

LABEL: VICTORIA: Etta's Glen, Black's Spur, Healesville, W.W. Watts 968 \& 968a, 21 Dec 1906 [5 packets in one specimen folder].

TYPES: G, NSW. 
COMMENT: The NSW specimen consists of 5 small packets variously labelled Watts 968, Watts 968a or Watts $968+968 a$. All are annotated 'Chiloscyphus maximus St. sp. n. 1913' in Watts' hand. Apparently a large variety of C. coalitus (Hook.) Nees in Gottsche, Lindenb. \& Nees (Scott 1985: 133).

Chiloscyphus montanus Stephani (Stephani \& Watts 1914: 103)

PRото: 'Australia (Neate's Glen, Blackheath): Watts, 927.'

LABEL: NEW SOUTH WALES: Rotunda, Neate's Glen, B[lack]heath, on damp ground base of cliff, W.W. Watts 927, 7 Apr 1903 [3 packets in one folder].

TYPES: G, NSW.

COMMENT: = Geocalyx caledonicus Stephani fide Grolle (1979).

Fimbriaria dioica Stephani (Stephani \& Watts 1914: 104)

PRoto: 'Australia, N.S. Wales (near Gladesville): Watts 1095.'

Label: New South Wales: off Pittwater Road, Gladesville, W.W. Watts 1095 E [J.F.] Shirley, Nov 1911 [3 specimens in one folder].

TYPES: G, NSW.

COMMENT: Fimbriaria is an illegitimate name but this taxon has not been formally transferred to Asterella, where it belongs. This group is under revision.

Fossombronia grossepapillata Stephani (1917: 73)

Proto: 'Australia (Watts legit.).'

LABEL: New SOUTH WaLES: park, Young, (mining holes), W.W. Watts 988, Sep-Oct 1906 [3 packets in one folder].

TYPES: G (22171), NSW.

Comment: G 22171 (Watts 988) designated as type specimen by Scott \& Pike (1988: 196).

Fossombronia integrifolia Stephani (1917: 73)

PRoto: 'Australia orientalis (N.S. Wales).'

Label: New South Wales: 1. Parramatta, E. Cheel (Hb Watts 799), 1 Oct 1910 [2 packets]; 2. Rookwood, Sydney, E. Cheel (Hb Watts 812), 5 Aug 1900 [2 packets].

TYPES: FH (812), G (22174), NSW.

COMMENT: Specimens of Watts 812 held at FH and G designated as the type by Scott \& Pike (1988: 197).

\section{Fossombronia wattsii Stephani (1917: 75)}

PRoTo: 'Australia, New South Wales (Watts legit.).'

Label: New South Wales: The Gap, near Young, W.W. W[atts] 881, 26 Sep 1905.

TYPES: G (Hb Levier 5155), NSW.

COMMENT: No locality or specimen number cited in protologue but $\mathrm{G}$ specimen of Watts 881 cited as type in Bonner (1965: 221). Type material (at NSW) checked and studied by Pike: 'abnormal spores present $8 / 3 / 82$ '. 
Frullania asperifolia Stephani (Stephani \& Watts 1914: 105)

PRoTo: 'Australia (Yarrangobilly Caves \& Mount Wilson): Watts 1093, etc).'

LABEL: New South WALES: 1. Yarrangobilly Caves, W.W. Watts 923, Jan 1906; 2. 'Yarrawa', Mt Wilson, W.W. Watts 1063, 10 Jan 1911;3. Denmarque, Mt Wilson, W.W. Watts 1078, 13 Jan 1911; 4. Falls beyond Kiandra road, Yarrangobilly Caves, W.W. Watts 926, Jan 1906; 5. Yarrangobilly [Caves], gully beyond hostel, W.W. Watts 922, Jan 1906; 6. Avenue, Mt Wilson, W.W. Watts 1093, 11 Jan 1911.

TYPES: G (18193), NSW.

COMMENT: Although two localities were cited in the protologue only one number (Watts 1093) was given and this is cited as the type by Bonner (1965: 239). Hattori (1979a) cites the type as 'G18193, the Avenue, Mt Wilson, $3500 \mathrm{ft}$ on tree Jan 11, 1911, leg. W.W. Watts (Hep. Aust. ex Herb. Watts $109[=1093]$ )' and considers this taxon conspecific with $F$. falciloba Taylor ex Lehm.

Frullania australis Stephani (1911: 659)

Proto: 'Australia orientalis, New South Wales.'

Label: New South Wales: E Ballina, tree, W.W. Watts 117, 14 Aug 1900 [3 packets in one folder].

TYPES: G (18194 ex Hb Levier 2638), NSW.

Comment: G 18194 is cited as the type by Bonner (1965: 241), without the Watts collection number. Known only from the type collection (Hattori 1979b: 123).

Frullania belmorensis Stephani (Stephani \& Watts 1914: 106)

Proto: 'Australia (Belmore Falls): Watts, 931.'

Label: New South Wales: Belmore Falls, W.W. Watts 931, 5 Oct 1908 [3 packets in one folder].

TYPES: G, NSW.

COMMENT: Known only from the type collection (Hattori 1979b: 126).

Frullania difficilis Stephani (1889: 160)

Proto: 'Australia, New South Wales.'

Label: New South Wales: Park, Gray's Island, Richmond River, W.W. Watts 125, 6 May 1900.

TYPES: G (ex Hb Levier 2632), MEL 61558, NSW.

COMMENT: No specimen number or specific locality is cited in the protologue but the type is cited by Bonner (1965: 288) as New South Wales, Richmond River, Pearce's Creek, 2 VIII 1900, W.W. Watts s.n., Hb Levier 2632 in Hb G'. Hattori (1987: 553) lists a number of other possible syntypes (including the above NSW specimen) and places F. difficilis in synonymy under Frullania (Trachycolea) seriata Gottsche ex Stephani.

Frullania elongata Stephani (1910: 423)

PRoto: 'Australia orientalis.'

Label: New South Wales: Pearce's Creek nr R. Trimble's, bark of old tree stump, W.W. Watts 96, 2 Aug 1900.

TYPES: G, NSW. 
COMMENT: Watts 96 is cited by Bonner (1965: 296) as the type. $=$ F . rubella fide Hattori (1979a: 354); = F. rubella Gottsche ex Stephani var. elongata (Stephani) Hattori fide Hattori (1983: 165).

Frullania excisula Stephani (Stephani \& Watts 1914: 107)

Proto: 'Australia, N.S. Wales (Mount Wilson and Blackheath): Watts 1030, 1071.'

LABEL: New SOUTH WALES: 1. Horseshoe Falls to Jungle, Blackheath, (rock by track), W.W. Watts 1030, 7 Jan 1911 [2 packets]; 2. Yarrawa, Mt Wilson, W.W. Watts 1071, 11 Jan 1911 [3 packets].

TYPES: G, MEL, NSW.

Comment: Bonner (1965: 229) cited Watts 1017 [= 1071] as the type. The NSW specimens of both Watts 1030 and 1071 are annotated by S. Hattori Apr. 1986: 'not type of $F$. squarrulosa but syntype of $F$. excisula'. $F$. excisula seems to be conspecific with Frullania falciloba (Hattori 1979a: 336).

Frullania ferdinandi-muelleri Stephani (1910: 417)

Proto: 'Australia, New South Wales.'

LABEL: NeW SOUTH WALES: Tintenbar(?), R[ichmond] R[iver], tea tree bark, W.W. Watts 153, 2 Aug 1900.

TYPES: G (18211 ex Hb Levier 2628), NSW.

COMmEnT: No information on locality, date, collector or specimen number(s) appears in the protologue but Hattori (1979a) describes this species as known only from the type collection and cites G 18211 as the type.

Frullania filipendula Stephani (1910: 419)

Proto: 'Australia, Queensland, New South Wales.'

LABEL: New SOUTH WaLes: E Ballina, base of saplings and on ground, heath, W.W. Watts 468, 17 June 1902 [2 packets in folder].

TYPES: G (18212 ex Hb Levier 3416), NSW.

COMMENT: No information on locality, date, collector or specimen number(s) appears in the protologue but Bonner (1965: 304) cites this specimen (G 18212) as the type. Seems to be conspecific with F. rubella (Hattori 1979a: 340).

Frullania howeana Stephani (Stephani \& Watts 1914: 107)

РRото: 'Lord Howe Island. (Watts 62).'

LABEL: NeW SOUTH WALES: near Face, east side of Mt Lidgbird, L[ord] H[owe] I[sland], W.W. W[atts] 62, 27 July 1911 [2 packets in folder].

TYPES: G (18215), NSW.

COMMENT: Endemic, known only from the type collection (Hattori 1979a: 346).

Frullania minutistipula Stephani (Stephani \& Watts 1914: 108)

Proto: 'Australia (Rodriguez Pass): Watts $1001^{\mathrm{b}}, 1001^{\mathrm{c}}$.'

Label: NeW South Wales: Rodriguez Pass, Blackheath, W.W. Watts $1001 b$ \& c, 4 Jan 1911. TYPES: G, NSW.

COMMENT: NSW specimen has been annotated (by Watts?): 'mixed with Frullania gracillima sp. n. and Radula wattsiana'. = F. rostrata (Hook. f. \& Taylor) Hook. f. \& Taylor ex Gottsche, Lindenb. \& Nees fide Hattori (1979a: 352). 
Frullania simmondsii Stephani (Stephani \& Watts 1914: 109)

Proto: 'Australia (near Brisbane): Simmonds leg. (Watts 1110).'

Label: QueEnsLAND: near Brisbane, J.H. Simmonds, Hb Watts 1110, 27 Aug 1887.

TYPES: G, NSW.

COMMENT: NSW specimen annotated as isotype by Hattori. Known only from the type collection (Hattori 1979b: 143).

Frullania subtropica Stephani (1910: 416)

Proto: 'Australia, New South Wales.'

Label: New South Wales: off Tintenbar road, 3 miles [5 km] from Ballina, Richmond River, tree, W.W. Watts 107, 30 June 1900.

TYPES: G (18230 ex Hb Levier 2602), NSW.

COMmEnT: Protologue does not include locality, date, collector or specimen information but G 18230 is cited as the type by Bonner (1965: 440).

Frullania variabilis Stephani (1910: 420)

Proto: 'Australia, New South Wales.'

Label: New South Wales: Cambewarra Mtn, W.W. W[atts] 628, 22 May 1903.

TYPES: G (ex Hb Levier 4416), NSW.

COMment: Protologue does not include locality, date, collector or specimen information but the above specimen in $\mathrm{G}$ (Hb Levier 4416) is cited as the type by Bonner (1965: 462). Known only from the type collection; probably a form of $F$. pycnantha (Hook. f. \& Taylor) Taylor ex Gottsche, Lindenb. \& Nees (Hattori 1979b: 149).

Haplomitrium intermedium Berrie (1963: 191)

PRoto: 'Between one and two miles along the road from Bilpin to Mount Irvine, Blue Mountains, N.S.W. HolotYPE: Sydney University Herbarium, Bryophyte No. 2/62. COTYPE: Professor R.M. Schuster Collection No. 50,616.'

LABEL: see below

TYPES: SYD (holotype; not located).

COMMENT: Probably on loan, but record not found.

Isotachis grandis Carrington $\mathcal{E}$ Pearson (1888: 1041)

Proto: 'On wet rocks, Lawson, Blue Mountains, June, 1884.'

LABEL: NEW SOUTH WALES: 1. Lawson, Blue Mountains, on wet rocks, [T. Whitelegge], June 1884; 2. Lawson, no 14 liverwort, [T. Whitelegge], June 1884.

TYPES: BM, MANCH?, NSW.

COMMENT: The former NSW specimen has been further annotated by Whitelegge: ' 17 Isotachis grandis n. sp. (small portion of stem) plXXVI 34 TW W.H.P.' and the latter has the additional information: ' 17 N. sp. No 34 P. Isotachis grandis'. Probably no. 34 of a batch sent to Pearson by Whitelegge.

Isotachis terricola Stephani (Stephani \& Watts 1914: 10)

Proто: 'Australia (Blackheath): Watts, 1016.' 
LABEL: New South Wales: short cut to Neate's Glen, Blackheath, creek, swamp, W.W. Watts 1016, 5 Jan 1911 [3 packets in folder].

TYPES: G, NSW.

COMment: $=$ I. intortifolia (Hook. f. \& Taylor) Gottsche fide Hatcher (1960: 602).

Jungermannia wattsiana Stephani (1917: 96)

Proto: 'Australia, New South Wales (Watts legit.)'

LABEL: NeW SOUTH Wales: 1 . Alst[onville] Cutting, 7 miles [11 km] nearly, W.W. Watts 303, 24 June 1901; 2. Alstonville Cutting, R[ichmond] R[iver], nearly 7 miles [11 km] from Ballina, W.W. W[atts] 303, 24 June 1901.

TYPES: G (14307 ex Hb Levier 3141), NSW.

Comment: No specimen numbers given in protologue but Bonner (1976: 410) cites the type as: 'NSW, Alstourith [= Alstonville] cutting, 7 miles from Ballina, 24.June.1901, Rev. Watts 303, Hb Levier 3141, Hb G 14307.'

Jungermannia whiteleggei Carrington \& Pearson (1888: 1051)

РRоTO: no specimens cited.

Label: New South Wales: Moore Park, [Sydney], [T. Whitelegge] 37, Dec 1884.

TYPES: K or MANCH?, NSW.

COMMENT: The NSW specimen has been annotated by Whitelegge: 'Jungermannia whiteleggei n. sp., pI XXXII 36 T.W. W.H.P.' It is probably no. 36 of a batch sent to W.H. Pearson. No specimen information is given in the protologue but the information on the packet is consistent with that found on other type specimens sent by Whitelegge to Pearson. = Chaetophyllopsis whiteleggei (Carrington \& Pearson) R. Schuster fide Schuster (1960: 69).

Lejeunea wildii Stephani (1889: 165)

Proto: 'Queensland, leg. C. Wild. - Herb. Brotherus.'

LABEL: QueEnSLAND: Hamilton, C.lJ.] Wild, July 1887 [2 identically labelled specimens]. TYPES: G (15847), BM, MANCH, MEL, NSW (H495 \& H496).

COMMENT: G 15847 cited as holotype and BM, MANCH and MEL as isotypes by Thiers \& Gradstein (1989: 6); almost certainly the only specimen matching the protologue. Reduced to synonymy under Acrolejeunea securifolia (Nees) Stephani ex Watts ssp. securifolia by Thiers \& Gradstein.

Lembidium dendroides Carrington \& Pearson (1888: 1047)

Proto: 'On wet earth, Mermaids Glen, Blackheath, Blue Mountains, 3,000ft. September 5, 1885.-T. Whitelegge.'

LABEL: New South Wales: Mermaids Glen, Blackheath, Blue Mts, on wet earth, [T. Whitelegge] 28, 5 Sep 1885 [2 similarly labelled specimens].

TYPES: BM, NSW (H170 \& H171).

COMMENT: NSW specimens annotated by Whitelegge: 'Lembidium dendroides n. sp., No. $16 \mathrm{P}^{\prime}$ and is probably no. 16 of a batch sent to Pearson. Although no collector's number is cited in the protologue all other label data are consistent with these NSW specimens being part of the type. = Kurzia dendroides (Carrington \& Pearson) Grolle fide Grolle (1963: 175). Still known only from the type (Schuster 1980: 394). 
Lepidozia appressifolia Stephani (1909: 583)

PRото: 'Australia orientalis (Watts); Tasmania (Moore)'

Label: New South Wales: Katoomba Falls, Blue Mountains, W.W. Watts 571, 15 Jan 1902. TYPES: G, NSW.

COMMENT: NSW specimen of Watts 571 is labelled 'TYPE' in Watts' handwriting.

Lepidozia buffalona Stephani (Stephani \& Watts 1914: 111)

Proto: 'Australia, (Buffalo Creek, near Gladesville; Blue Mountains and Cambewarra): Watts 977, 941, 978, 965, 982, 914 etc).'

Label: New South Wales: 1. Centennial Glen, B[lack]heath, W.W. Wlatts] 1045, 11 Jan 1911; 2. Horseshoe Falls, Blackheath, wet cliffs, W.W. W[atts] 1025, 7 Jan 1911; 3. Rotunda, Blackheath, W.W. Watts 1011, 4 Jan 1911 [3 packets]; 4. Cambewarra Mtn, damp places under cliffs above Baldys, W.W. W[atts] 914, Oct 1907.

TYPES: G, NSW.

COMMENT: Icones Hep. Steph. 5076 is based on Watts 977 (Geissler \& Bischler 1985: 21).

Lepidozia communis Stephani (Stephani \& Watts 1914: 111)

Proto: 'Australia, (Grand Canyon, Blackheath): Watts, 1014.'

Label: New South Wales: Grand Canyon, Blackheath, W.W. Watts 1014, 4 Jan 1911 [3 packets in folder].

TYPES: G, NSW.

COMMENT: Name in current use.

Lepidozia crassitexta Stephani (Stephani \& Watts 1914: 111)

Proto: 'Australia, (Rodriguez Pass, Blackheath): Watts, 1005.'

LABEL: NeW SOUtH Wales: Rodriguez Pass, Blackheath, W.W. Watts 1005, 4 Jan 1911 [3 packets in folder].

TYPES: G, NSW.

COMMENT: Name in current use.

Lepidozia furcatifolia Stephani (Stephani \& Watts 1914: 112)

Proto: 'Australia, (Horse Shoe Falls, Blackheath): Watts, 1027.'

LABEL: NeW SOUTH WALES: 1. Horse Shoe [Horseshoe] Falls, Blackheath, wet cliff, W.W. Watts 1027, Jan 1911; 2. Horse Shoe [Horseshoe] Falls, Blackheath, wet cliff, W.W. Watts $1027 b$, Jan 1911 [2 packets].

TYPES: G, NSW (Watts 1027, also? 1027b).

COMMENT: The NSW specimen of W.W. Watts $1027(b)$, which is labelled 'TYPE' (probably Watts' handwriting), may also be part of the type.

Lepidozia gracillima Carrington $\mathcal{E}$ Pearson (1888: 1045)

PRoto: 'on wet rocks, Botany Bay, June, 1885'

LABel: New South Wales: 1. Botany Bay, on wet rocks, [T. Whitelegge 24], June 1885;

2. Botany Bay, on wet rocks, [T. Whitelegge 24], June 1885.

TYPES: BM or MANCH?, NSW. 
COMMENT: The former NSW specimen has been annotated by Whitelegge: 'Lepidozia gracillima n. sp. plXXVII, 51 TW. W.H.P.' and the latter: ' 24 N. sp. No 51.P. Lepidozia gracillima'. Probably no. 51 of a batch sent to Pearson by Whitelegge.

Lepidozia hastatistipula Stephani (Stephani \& Watts 1914: 113)

Proto: 'Australia, (Healesville, Vict.): Watts, 966.'

LABEL: Victoria: 'Airlee', Healesville, (by summer house), W.W. Watts 966, 22 Dec 1906 [2 similarly labelled packets in specimen folder].

TYPES: G, NSW (H159).

COMMENT: Name in current use.

Lepidozia lateconica Stephani (Stephani \& Watts 1914: 113)

PRoTo: 'Australia, (Barron Falls, North Queensland; leg Mrs Brotherton) Watts, 976.'

Label: QueENSLAND: 1. Kuranda, Mrs Brotherton, W.W. Watts 976, June 1910 [3 similarly labelled packets in folder]; 2. Kuranda, Barron Falls, Mrs Brotherton, W.W. Watts 976, June 1910 (NSW H160).

TYPES: G, NSW.

COMMENT: = Kurzia lateconica (Stephani) Grolle fide Grolle (1963: 175). Apparently known only from the type (Schuster 1980: 387).

Lepidozia microstipula Stephani (Stephani \& Watts 1914: 114)

PRoTo: 'Australia, (Lane Cove River, above Fig Tree Bridge): Watts, 958.'

LABEL: New SOUTH WALES: 1. Lane Cover River, under rocks, old residential area, first wharf above Fig Tree, W.W. Watts 958, Nov 1910; 2. Near Lane Cove River, above Hunters Hill, under rock in old residential area, W.W. Watts 958, Nov 1910 [2 packets in folder: NSW H161].

TYPES: G, NSW.

COMMENT: Name in current use.

Lepidozia multifida Stephani (Stephani \& Watts 1914: 114).

Proto: 'Australia, New South Wales (Blue Mountains): Watts, 974, 975, 964.'

Label: New South Wales: 1. Water Nymphs Dell, Wentworth Falls, W.W. Watts 974, 3 Jan 1910; 2. Nymphs Dell, Wentworth Falls, W.W. Watts 975, 3 Jan 1910 [3 packets in folder, 2 mistranscribed as Jan 1911]; 3. Loc?, [W.W. Watts] 964, 1910.

TYPES: G, NSW.

COMMENT: Name in current use.

Lepidozia nova Stephani (Stephani \& Watts 1914: 115)

РRото: 'Australia, (Blue Mountains and Mount Wilson): Watts, 1033, 1054 and 1066.'

LABEL: New SouTh Wales: 1. Mermaids Glen, Blackheath, W.W. Watts 1054, 3 Jan 1911; 2. Mermaids Glen, Blackheath, W.W. Wlatts] 1054, 3 Jan 1911 (NSW H162); 3. Track from Horse Shoe [Horseshoe] Falls to Jungle, Blackheath, W.W. Watts 1033, Jan 1911; 4. Horse Shoe [Horseshoe] Falls to jungle, Blackheath, W.W. W[atts] 1033, 7 Jan 1911 (NSW H163); 5. 'Yarrawa', Mt Wilson, W.W. Watts 1066, 11 Jan 1911 [2 packets]. TYPES: G, NSW.

Comment: Name in current use. 
Lepidozia quadriseta Stephani (1909: 582)

Proto: 'N. South Wales (Watts).'

LabEL: New SOUTH WALES: 1. Horseshoe Falls, Blackheath, W.W. Wlatts] 1027, 7 Jan 1911

(NSW H164); 2. E Ballina, swamp, heath, W.W. Watts 433, 11 June 1902.

TYPES: G, NSW.

COMMENT: NSW H164 has been annotated 'type' by Watts. = Telaranea tetradactyla (Hook. f. \& Taylor) E. Hodgson fide Grolle (1963).

Lepidozia quadristipula Stephani (Stephani \& Watts 1914: 115)

Proto: 'Australia, (Rotunda, Neate's Glen, Blackheath): Watts 1009.'

Label: New South Wales: 1. Rotunda, Blackheath, W.W. Watts 1009, 4 Jan 1911; 2. Rotunda, Blackheath, W.W. Watts 1009, 4 Jan 1911 (NSW H167).

TYPES: G, NSW.

COMMENT: Name in current use.

Lepidozia septemfida Stephani (1909: 588)

Proto: 'Australia, N.S. Wales (Watts).'

LabeL: New SOUtH Wales: Katoomba Falls, W.W. Watts 574, 15 Jan 1902.

TYPES: G, NSW (H212).

COMmENT: NSW specimen annotated by Watts: 'Co-type. Mixed with L. appressifolia.' Name in current use.

Lepidozia tripilosa Stephani (Stephani \& Watts 1914: 116)

Proto: 'Australia, (Centennial Glen, Blackheath): Watts, 1043.'

Label: New South Wales: Centennial Glen, Blackheath, W.W. Watts 1043, 10 Jan 1911 [2 similarly labelled packets in specimen folder].

TYPES: G, NSW.

COMMENT: Icones Hep. Steph. n. 5100 is based on Watts 1043 (Geissler \& Bischler 1985: 47).

Leptocolea wattsiana Stephani (1916: 856)

Proto: 'Australia. New South Wales.'

LABEL: NeW SOUTH WaLES: 1. nr. beach, H. C[ree]k, [W.W. Watts] 232, Mar 1901; 2. on tree by J.W. Sharpe's sliprails, nr. beach, above Ballina, W.W. Watts 232, Mar 1901 [both specimens in same folder].

TYPES: G, NSW.

COMMENT: Specimen 1 above further annotated by Watts: 'Cololejeunea wattsiana sp. $\mathrm{n}$. in letter from Dr. Levier July ' 02 '. It has not been possible to identify the locality of 'H. Ck' or 'J.W. Sharpe's sliprails'. Specimen of Watts 232 at G cited as holotype and NSW specimen as isotype by Thiers (1988: 123) who does not indicate if the G specimen has been labelled as the type by Stephani. Reduced to synonymy under Cololejeunea lanciloba Stephani by Thiers. 
Marchantia paludicola Stephani (Stephani \& Watts 1914: 120)

Proto: 'Australia, New South Wales, (Cambewarra): Watts, 1106, 1102.'

Label: New South Wales: 1. above Baldys, Cambewarra Mountain, W.W. W[atts] 1106, 18 Oct 1907; 2. above Baldys, Cambewarra Mountain, [W.W.] Watts 1106, Oct 1907; 3. In swamp, $3900 \mathrm{ft}$ [1170 m], Mt Werong, R.H. Cambage, 4 Dec 1911, Hb Watts No. 1102.

TYPES: G, NSW.

COMMENT: NSW specimen collected by R.H. Cambage has been further annotated 'cotype' whilst the other two NSW specimens have been annotated 'type' by Watts. = Marchantia berteroana Lehm. \& Lindenb. fide Bischler-Causse (1989: 82). NSW specimens cited as syntypes by Bischler-Causse.

Marchantia wattsiana Stephani ex Bonner (Bonner 1953: 112)

Рвото: 'Australia: New South Wales, on stone in Whian Creek, Federal, Richmond River, 13 Aug 1902, Watts, s.n. (Hb Levier 4529), lectotype; Miami [= Minmi] July 1901 Watts 317 (Hb Levier 3116 bis); Richmond River, 1901, Watts 308 (Hb Levier 3111).'

LABEL: New SOUth WALES: 1. Whian Whian Creek, Federal, Richmond River (above Crossing), on stone, W.W. Watts 512, 13 Aug 1902; 2. Richmond River, probably Shaw's Bay, W.W. Watts 308, 1901; 3. loc.?, perhaps Minmi, W.W. Watts 317, July 1901.

TYPES: G (ex Hb Levier 4529: lectotype), NSW.

COMMENT: In 1953 Bonner published a Stephani manuscript, adding to the protologue a lectotype and list of specimens based on Stephani's herbarium at G; he did not examine Watts' collections held at NSW. = Marchantia pileata Mitten fide BischlerCausse (1989: 119) who considers Stephani's description to be based, at least partly, on Watts 308.

Mastigobryum corbieri Stephani (Stephani \& Watts 1914: 122)

Proto: 'Australia, (Kingwell, Wyong): Watts, 971'.'

LABEL: NeW SOUTH WALES: gully south of house, 'Kingwell', Wyong, W.W. Watts 971b, Nov 1907 [2 similarly labelled specimens].

TYPES: G, NSW (H286 \& H301).

COMMENT: Mastigobryum is an illegitimate name but this taxon has never been formally transferred to Bazzania, where it belongs (Scott \& Bradshaw 1986: 67). Watts 971a is the type specimen of Balantiopsis subkingwella, see above.

\section{Mastigobryum dentistipulum Stephani (Stephani \& Watts 1914: 122)}

Proto: 'Australia, New South Wales (Lane Cove River and Valley of Waters): Watts 1096, 1129, 1104.'

Label: New South Wales: 1. Lane Cove River, W.W. Watts 1096, May 1912 (NSW H287);

2. Valley of Waters [Wentworth Falls], W.W. Watts 1129, [3 packets in folder: NSW H288]; 3. Lane Cove River, W.W. Watts 1104, May 1912.

TYPES: G, NSW.

COMMENT: NSW H287 \& H288 have been annotated 'co-type' and 'type' respectively by Watts. Mastigobryum is an illegitimate name but this taxon has never been formally transferred to Bazzania, where it belongs (Scott \& Bradshaw 1986: 67). 
Mastigobryum erosifolium Stephani (Stephani \& Watts 1914: 122)

PRoto: 'Australia (Cambewarra Mountain): Watts legit, 917.'

Label: New South Wales: 1. Damp places under cliff, Cambewarra Mountain, W.W. Watts 917, Oct 1907; 2. Under cliff, above Baldy's, Cambewarra, W.W. Watts 917, Oct 1907.

TYPES: G (15074), NSW (H302 \& H289).

COMMENT: = Acromastigum colensoanum (Mitten) Evans ex Reimers fide Grolle (1972: 547).

Mastigobryum gracillimum Stephani (Stephani \& Watts 1914: 123)

Proto: 'Australia, New South Wales, (Wyong and Valley of Waters): Watts, 939, 987.'

Label: New South Wales: 1. Gully south of house, 'Kingwell', Wyong, W.W. Watts 939, Nov 1907 [2 specimens in separate folders: original Watts packet is NSW H291]; 2. S Wyong, W.W. Watts 987, 1909 (NSW H292); 3. Valley of Waters [Wentworth Falls], W.W. W[atts] 1130, Sep 1912 (NSW H290) [3 packets in folder].

TYPES: G, NSW.

COMMENT: The NSW specimens of Watts 1130 are annotated: 'Mastigobryum gracillimum St. sp. n. Jan 1913' (the specimens of Watts $939 \mathcal{E} 987$ are similarly labelled but lack the 'sp. n.' designation). Mastigobryum is an illegitimate name but this taxon has never been formally transferred to Bazzania, where it belongs (Scott \& Bradshaw 1986: 67).

Mastigolejeunea wattsiana Stephani (1912: 780)

PRoTo: no specimens cited.

LABEL: New South Wales: Wallace's Hill, Possum Shoot, R[ichmond] R[iver], on vine, W.W. W[atts] 350, Oct 1901.

TYPES: FH, G (21835), NSW.

COMmENT: G 21835 (Watts 350) cited as holotype and FH as isotype by Thiers \& Gradstein (1989: 50) who do not indicate if the G specimen has been labelled as the type by Stephani. Reduced to synonymy under Mastigolejeunea ligulata (Lehm. \& Lindenb.) Schiffner by Thiers \& Gradstein.

Metzgeria howeana Stephani (Stephani \& Watts 1914: 126)

PROTO: 'Australia, Mount Wilson and Wyong: Watts legit 1065.'

Label: New South Walfs: 1. 'Yarrawah', Mt Wilson, W.W. W[atts] 1065, 11 Jan 1911; 2. gully over mountain SW of 'Kingwell', Wyong, W.W. Watts 948, 24 Aug 1910.

TYPES: G, NSW.

COMMENT: There are four other NSW collections from Yarrawa (Watts 1058, 1061, 1069 E 1072), all dated 11 Jan 1911 and annotated 'co-type'. The NSW specimen from Wyong is annotated 'type' in Watt's hand. This species is not listed in Scott \& Bradshaw (1986); name in current use.

Pallavacinia campanulata Stephani (Stephani \& Watts 1914: 127), as 'Pallavacinius' Proto: 'Australia, New South Wales, (Valley of Waters): Watts legit, 1111.'

LABEL: New SOUTH WaLES: Valley of Waters [Wentworth Falls], [W.W.] Watts 1111, Sep 1912 [2 packets in folder]. 
TYPES: G, NSW (H224).

COMMENT: Name in current use.

Physocolea casuarinae Stephani (1916: 909)

PROTO: 'Australia orientalis. New South Wales.'

LABEL: New South WALES: 1. Fig Tree, Lane Cove R[iver], [Sydney], on Casuarina, W.W. W[atts] 792, 1903; 2. bottom of gully above bridge, Fig Tree, Lane Cove River, on Casuarina, [W.W.] Watts 792, 1903 [both specimens are in the same folder].

TYPES: G (17524), NSW.

Comment: G 17524 (Watts 792) cited as holotype by Thiers (1988: 134) who does not indicate if the $G$ specimen is labelled as the type by Stephani. Reduced to synonymy under Cololejeunea minutissima (Smith) Schiffner by Thiers.

Plagiochasma queenslandicum Stephani (1917: 9)

Proto: 'Australia, Queensland (Simmonds legit).'

Label: QueEnsland: ex Hb Simmonds, Watts Q258, Aug 1887.

TYPES: G, NSW.

COMMENT: = Reboulia hemisphaerica fide Bischler (1979: 76). Recently reinstated as $\mathrm{R} e^{-}$ boulia queenslandica (Stephani) Hicks fide Hicks (1992: 114) who cites the type as $\mathrm{nr}$ Brisbane, J.H. Simmonds 16340, 1887, Herb. Watts 611 (4490) (G, FI [FH]).

Plagiochila rossii Stephani (Stephani \& Watts 1914: 130)

Proto: 'Australia, N.S. Wales (The Jungle, Blackheath): Watts 1035.'

LABEL: New SOUTH WaLEs: Blackheath, The Jungle, W.W. Watts 1035, 7 Jan 1911 [3 packets in folder].

TyPes: G, NSW (H344).

COMMENT: = Plagiochila baileyana Stephani fide Inoue (1986: 377).

Plagiochila serrifolia Stephani (Stephani \& Watts 1914: 130

PRото: 'Australia, New South Wales (Wyong): Watts, 1100.'

LABEL: NeW South WaLES: gully over mountain SW of 'Kingwell', Wyong, [W.W.] Watts 1100, 23 Aug 1910 [2 packets in folder].

TYPES: G, NSW (H345).

COMMENT: = Plagiochila vitiensis Mitten fide Inoue (1986: 364).

Plagiochila victoriae Stephani (Stephani \& Watts 1914: 131)

PRoto: 'Australia, Victoria (Lorne: leg. Miss E.L. Watts) Hb Watts, 910.'

LABEL: Victoria: Lorne, leg. Ethel Watts, Hb W.W. Watts 910, Dec 1909.

TYPES: G, NSW (H370).

COMMENT: = Plagiochila baileyana fide Inoue (1986: 377). 
Plagiochila wattsii Stephani (in Rodway 1918: 105)

Proto: 'On Fagus log, Pioneer track, Blue Tier (Weymouth). Determined by Stephani.' LABEL: New South Wales: Pearce's Creek, Richmond River, on tree, W.W. Watts 19, 19 June 1900.

TYPES: G (ex Hb Levier 2306), NSW.

COMment: Rodway (1918: 105) reported this species from Tasmania, attributing the species description and identification to Stephani. In 1921 Stephani redescribed Plagiochila wattsii as a new species (the protologue cites the type specimen as: 'Australia. New South Wales (Watts legit)'. The Stephani description does not match the one published in Rodway. Inoue \& Schuster (1971) were unable to locate Rodway's specimen, and they suggested that it is probably a species of Lophocolea. The G specimen of Watts 19 is cited as the type by Inoue \& Schuster (1971: 221) and Inoue (1986: 371). Further work to clarify the status of this taxon is required!

Porella crawfordii Stephani (1889: 270), as 'cranfordi'

PRото: ‘New England, Moona near Walcha leg. A. R. Cranford 1884....Queensland, Toowoomba, Hartmann 1876, Queensland F. M. Bailey (Herb. Brotherus Nr. 11). N. S. Wales Rev. Dr. Woolls. 1884'

Label: New South Wales: Moona near Walcha, New England, A.R. Crawford, 1884.

TYPE: G (lectotype), NSW (H259: isolectotype).

CommEnT: Lectotypified by Hattori (1985: 53). This species was named after its collector, A.R. Crawford, but Stephani appears to have misread the handwritten label. Stephani's spelling, although original, must count as an orthographic error and was amended by Scott \& Bradshaw (1986: 79).

Riccardia babindae Hewson (1970b: 85)

PROTO: 'Holotype-The Boulders, Babinda, N. Qld., Hewson 460, viii.1964 (NSW): IsOTYPE (BRI).'

Label: QueEnsland: Babinda, The Boulders, H.J. Hewson H64.79.460, 26 Aug 1964.

TYPE: NSW (179242: holotype), BRI (isotype).

COMMENT: Name in current use.

Riccardia bliklika Hewson var. bliklika (Hewson 1970b: 83)

PRoto: 'Holotype-Vision Falls, Lake Eacham, Atherton Tableland, N. Qld., on weathering basalt in rainforest, Hewson, 387, 8.1964, (NSW): IsOTYPE (BRI).'

Label: QueEnsland: Lake Eacham, Vision Falls, H.J. Hewson H64.68.387, 20 Aug 1964.

TYPES: NSW (179243: holotype), BRI (isotype).

COMMENT: Name in current use.

\section{Riccardia hypipamensis Hewson (1970b: 97)}

Proto: 'Holotype- The Crater, Mt. Hypipamee, N. Qld, on weathering basalt in rainforest, Hewson, 448, 8.1964 (NSW): IsOTYPE (SYD).'

Label: QueEnSLAND: Mt Hypipamee, The Crater, H.J. Hewson H64.76.448, 24 Aug 1964.

TYPES: NSW (holotype), SYD (isotype-not located).

COMMENT: Name in current use. 


\section{Riccardia macdonaldiana Hewson (1970b: 93)}

PRoto: 'Holotype- Mossman Gorge, 3/4 mile beyond Mossman Mission, on soil and roots in rainforest, Hewson 326 [= 362], 8.1964, (NSW): IsOTYPE (BRI).'

LABEL: North QueENSLAND: about $3 / 4$ mile $[1 \mathrm{~km}]$ beyond Mossman Mission, Mossman Gorge-Creek, soil and roots, rainforest, granite, H.J. Hewson H64.66.362, 19 Aug 1964.

TYPES: NSW (holotype), BRI (isotype).

COMMENT: In the protologue the number is given as 326 , a typographical error for the correct number 362 .

\section{Riccardia minima Carrington $\mathcal{E}$ Pearson (1888: 1055)}

Proto: 'On the surface of peat, in bogs, Coogee Bay, New South Wales, May, 1885.'

Label: New South Wales: Coogee Bay, in flower, [T. Whitelegge 45], May 1885.

TYPES: MANCH (Kk854), BM, NSW (H247).

COMMENT: NSW specimen further annotated by Whitelegge: ‘45 N. sp. Riccardia minima No. 37.' i.e. probably no. 37 of a batch sent to Pearson by Whitelegge. MANCH Kk854 cited as holotype by Hewson $(1970 b: 84) .=$ Riccardia lobulata (Colenso) E. Hodgson fide Brown \& Braggins (1989: 43).

Riccia areolata $\mathrm{Na}$-Thalang (1980: 78)

PRoto: 'Holotype: New South Wales, Prospect Reservoir, Na-Thalang 236, 15.vi.1967 (NSW).'

LABEL: New SOUTH WALES: Prospect Reservoir, open grassland at roadside, O. Na-Thalang 236, 15 June 1969.

TYPES: NSW (M11480: holotype), SYD (isotype-not located).

COMMENT: Name in current use.

Riccia asprella Carrington $\mathcal{E}$ Pearson (1888: 1039)

Proto: 'On earth, Head of Double Bay, Sydney, July, 1885.'

Label: New South Wales: Head of Double Bay, on earth, [T. Whitelegge 50], June 1885.

TYPES: BM or MANCH?, NSW (H94).

COMMENT: The NSW specimen has been further annotated by Whitelegge: ' 50 N. sp., Riccia asprella, No 53.P.'; probably no. 53 of a batch sent to Pearson. NSW specimen cited as holotype by Na-Thalang (1980: 84 ) but the original specimen is almost certainly held at MANCH or BM.

Riccia blackii Na-Thalang (1980: 81)

Proto: 'Holotype: Central Australia, Mt Olga. D. Black (Na-Thalang 87), 11.viii[vi].1966 (NSW); ISOTYPE (SYD).'

LABEL: NORTHERN TERRITORY: top of Mt Olga, along creek bank, D. Black, O. Na-Thalang 87, 11 June 1966.

TYPES: NSW (M11478: holotype), SYD (isotype).

COMMENT: Name in current use. 
Riccia bullosa Link var. vesiculosa Carrington $\mathcal{E}$ Pearson (1888: 1058)

Proto: 'On earth, Parramatta, August, 1885.'

Label: New Soutu Wales: 1. Parramatta, on earth, [T. Whitelegge 49], Aug 1885 (NSW H87); 2. Parramatta North, on earth, [T. Whitelegge 49], 16 Aug 1885; 3. Parramatta (North), on earth, [T. Whitelegge 49], 16 Aug 1885 (NSW H254).

TYPES: BM or MANCH?, NSW.

COMment: The NSW specimens are further annotated by Whitelegge: '49, 21 T.W. W.H.P', '49, No. 21, Riccia bulbosa var. vesiculosa' and 'No. 49 C\&P, No. 24W.' respectively. No collector or number are cited in the protologue but the other data and annotations are consistent with the NSW specimens being part of the type. Probably no. 21 of a batch sent to Pearson. Na-Thalang (1980: 116) cites the type as 'Holotype: New South Wales, Parramatta, Whitelegge 8, 1885 (NSW H87); isotype (SYD).' No material was located at SYD and the original specimen is almost certainly held at MANCH or BM. = Riccia vesiculosa (Carrington \& Pearson) Stephani fide Na-Thalang (1980: 114).

Riccia caroliniana $\mathrm{Na-Thalang} \mathrm{(1980:} \mathrm{72)}$

Proto: 'Holotype: Robin Water Fall, N.T., Na-Thalang 288 28.i.1969 (NSW); ISOTYPE (SYD).'

LABEL: NORTHERN TERRITORY: c. 2 miles [3 km] from Robin Waterfall, creek bank among grass, O. Na-Thalang 28828 Jan 1969.

TYPES: NSW (holotype; not located), SYD (isotype).

COMMENT: Probably on loan.

Riccia collata Na-Thalang (1980: 122)

Proto: 'Holotype: New South Wales, Tunmallallee Na-Thalang 220 26.iii.1967 (NSW); ISOTYPE (SYD).'

LABEL: New South WaLeS: Tunmallallee, along creek bank, O. Na-Thalang 220, 26 Mar 1967.

TYPES: NSW (M11478: holotype), SYD (isotype).

CoMment: Name in current use.

Riccia duplex Lorbeer var. megaspora $\mathrm{Na-Thalang} \mathrm{(1980:128)}$

Proto: 'HolotyPE: Northern Territory, East Alligator River, R.C. Carolin 6880 15.v.1968 (NSW).'

LABEL: NORTHERN TERRITORY: margins of lagoon, East Alligator River, about 7 miles [11 km] S Cahills Crossing, R.C. Carolin 6880, 16 May 1968.

TYPE: NSW (holotype).

COMMENT: Name in current use.

Riccia longiciliata Na-Thalang (1980: 75)

Proto: 'Holotype: Northern Territory, Palm Valley, Na-Thalang 100A, 15.viii.1966 (NSW); ISOTYPE (SYD).'

Label: Northern Territory: Palm Valley, open sandy soil or on pocket of rocks, grow[ing] mix[ed] with R. albida, O. Na-Thalang 100A, 15 Aug 1966. 
TYPES: NSW (M11477: holotype), SYD (isotype-not located).

COMMENT: Name in current use.

Riccia luticola Na-Thalang (1980: 123)

PRoto: 'Holotype: Northern Territory, c. 5 miles south of Katherine. Na-Thalang 321, 5.ii.1969 (NSW).'

LABEL: NORTHERN Territory: c. 5 miles $[8 \mathrm{~km}$ ] south of Katherine, margin of swamp, among grass, O. Na-Thalang 321, 5 Feb 1969.

TYPE: NSW (2 specimens labelled holo- and isotype respectively).

COMMENT: Name in current use.

Riccia marginata Carrington \& Pearson (1888: 1056)

PRoto: 'Parramatta, N.S.W., August 1885.'

Label: New South Wales: 1. Parramatta North, on earth, [T. Whitelegge 48], 16 Aug 1885;

2. Parramatta, on earth, [T. Whitelegge 48], Aug 1885.

TYPES: BM or MANCH?, NSW (H89 \& H91), SYD.

COMMENT: The latter NSW specimen is further annotated by Whitelegge: " 48 Riccia marginata n. sp., plXXXV, 20 TW WHP.' i.e. probably no. 20 of a batch sent to Pearson. Na-Thalang (1980: 100) cites NSW H89 as the holotype and NSW H91 and SYD as isotypes but there is almost certainly a BM or MANCH specimen which would have been the material seen by Pearson.

Riccia multifida (Stephani) Stephani var. filiformis Na-Thalang (1980: 131)

PRoto: 'Holotype: New South Wales, Coonabarabran, Mallallee Creek, O. Na-Thalang 215, 26.iii.1967 (NSW).'

Label: New SOUTH Wales: along the Mallallee Creek, Coonabarabran, O. Na-Thalang 215, 26 Mar 1967 [2 similarly labelled specimens in separate folders].

TYPE: NSW (holotype).

COMMENT: Name in current use.

Riccia multifida (Stephani) Stephani var. torticolla Na-Thalang (1980: 134)

Proto: 'Holotype: Western Australia, Poison Creek, 50 miles north of Leonora, R.C. Carolin 5834, Aug 1966 (NSW).'

Label: Western Australia: Poison Creek, 50 miles [80 km] N of Leonora, mud at edge of water hole, R.C. Carolin 5834, Aug 1967.

TYPE: NSW (holotype).

COMMENT: Name in current use.

Riccia olgensis $N a-$ Thalang (1980: 100)

Proto: 'HolotyPe: Central Australia, Mt Olga, D.R. Selkirk, (Na-Thalang 136 [= 135]), 20 Aug 1966 (NSW); ISOTYPE (SYD).'

LABEL: NORTHERN TerRitory: on top of Mt Olga, along creek bank, D.R. Selkirk (O. NaThalang 135), 20 Aug 1966.

TYPES: NSW (M11479: holotype), SYD (isotype).

COMMENT: Error in protologue; the number on the NSW and SYD specimens is 135 not 136. 
Riccia rorida $N a-$ Thalang (1980: 101)

Proto: 'Holotype: A.C.T., Cotter R., Na-Thalang 191, 27.i.1969 (NSW).'

LABEL: AUSTRALIAN CAPITAL TERrITORY: Cotter River, open grassland along the river bank, O. Na-Thalang 191, 27 Jan 1969.

TYPE: NSW (holotype).

COMMENT: Name in current use.

Riccia spongiosula Na-Thalang (1980: 113)

Proto: 'Holotype: New South Wales, Jubbal, W.W. Watts 144 [= 744], 22.vii.1903 (NSW H97).'

LABEL: New SOUTH WALES: 1. near Tubbul, Young, ground among rocks, W.W. W[atts] 744, 22 July 1903; 2. near Tubbul, Young, ground among rocks, (this was prob[ably] opp[osite] Fowlers), [W.W. Watts] 744, 22 July 1903.

TYPES: NSW (H97: holotype; 244529: isotype).

COMMENT: Both of the NSW specimens are annotated: 'Riccia spongiosa Steph. sp. n. ms. 1906 ' in Watt's hand. Na-Thalang appears to have made three typographic errors in the protologue but all the other information available (Na-Thalang 1980: 114) suggests that these are the correct specimens.

Symphyogyna interrupta Carrington E Pearson (1888: 1053)

PRoTo: no specimens cited.

LABel: New South Wales: 1. Mossmans Bay, [near Sydney], wet rocks, [T. Whitelegge 42], 8 Aug 1885; 2. Mossmans Bay, [near Sydney], [T. Whitelegge 42], 8 Aug 1885.

TYPES: BM or MANCH?, NSW (2 specimens).

COMMENT: NSW H360 has been annotated by Whitelegge: 'Symphyogyna interrupta $\mathrm{n}$. sp., plXXXIII, 55 T.W. W.H.P.' and NSW H361 as 'n. sp., No 55P.' Presumably specimen no. 55 of a batch sent to Pearson. No specimen information is given in the protologue and the type material at BM or MANCH has not been sighted but the information on the packet is consistent with that found on other type specimens sent by Whitelegge to Pearson.

\section{Symphyogyna multiflora Stephani (1917: 68)}

Рвото: 'Australia. New South Wales (Watts legit.).'

LABEL: New South Wales: Valley of Waters [Wentworth Falls], W.W. Watts 1127, Sep 1912.

TYPES: G, NSW.

COMMENT: The NSW specimen is further annotated: 'Symphyogyna multiflora St. sp. nov. det Jan.1913 (type)' in Watts' hand.

Trichocolea minutifolia Stephani (Stephani \& Watts 1914: 134)

Proto: 'Australia (Wyong): Watts legit, 950.'

LABEL: NEW SOUTH WALES: gully over mountain, SW of 'Kingwell', Wyong, W.W. Watts 950, 23 Aug 1910 [4 packets in one folder].

TYPES: G, NSW (H80).

COMMENT: Name in current use. 
Trichocolea wattsiana Stephani (Stephani \& Watts 1914: 135)

РRото: 'Australia (Wyong): Watts, 986.'

Label: New South Wales: 1. Gully beyond mountain SW of 'Kingwell', Wyong, W.W. Watts 946, Aug 1910; 2. Gully over mountain SW of 'Kingwell', Wyong, W.W. Watts 946, 23 Aug 1910 [3 packets in one folder].

TYPES: G, NSW (H75 \& H257).

COMMENT: Watts 986 in the protologue is probably a typographic error for 946 . The former NSW specimen (H257) has been further annotated: 'Trichocolea wattsiana St. sp. n. 1913, Type' in Watts' hand.

\section{Acknowledgements}

P. Geissler kindly checked data sent to Geneva against specimens examined by Stephani in preparing descriptions, to assist with designation of status of types held in Sydney. H.J. Hewson checked data based on her studies.

\section{References}

Bastow, R.A. (1888) Tasmanian Hepaticae. Pap. \& Proc. Roy. Soc. Tasmania for 1887: 209-289.

Bastow, R.A. (1914) Victorian hepaticae. Victorian Nat. 31(5): 74-81.

Berrie, G.K. (1963) Australian liverworts I. Haplomitrium intermedium sp. nov. (Calobryales). Proc. Linn. Soc. New South Wales 87: 191-195.

Bischler, H. (1979) Plagiochasma Lehm. et Lindenb. III. Les taxa d'Asie et d'Océanie. J. Hattori Bot. Lab. 45: 25-79.

Bischler-Causse, H. (1989) Marchantia L. The Asiatic and Oceanic taxa. Bryophytorum Bibliotheca 38: 1-317.

Bonner, C.E.B. (1953) De Hepaticis II. An unpublished section of volume 6 of Stephani's Species Hepaticarum: the genus Marchantia. Candollea 14: 101-12.

Bonner, C.E.B. (1965) Index Hepaticarum. Part V. Delavayella to Geothallus. (Cramer: Weinheim)

Bonner, C.E.B. (1976) Index Hepaticarum. Part VIII. Jungermannia. (Cramer: Vaduz)

Brown, E.A. \& Braggins, J.E. (1989) A revision of the genus Riccardia S.F. Gray in New Zealand with notes on the genus Aneura Dum. J. Hattori Bot. Lab. 66: 1-132.

Carrington, B. \& Pearson, W.H. (1888) List of Hepaticae collected by Mr Thomas Whitelegge in New South Wales, 1884-5. Proc. Linn. Soc. New South Wales ser. 2, 2(4): 1035-1060.

Downing, A.M., Ramsay, H.P. \& Schofield, W.B. (1991) Mosses in the vicinity of Jenoloan Caves. Cunninghamia 2: 371-384.

Engel, J.J. (1968) A taxonomic monograph of the genus Balantiopsis (Hepaticae). Nova Hedwigia 16 (1-2): 83-130.

Geissler, P. \& Bischler, H. (1985) Index Hepaticarum. Vol. 10. Lembidium to Mytilopsis. (Cramer: Vaduz)

Grolle, R. (1963) Ueber Kurzia v. Martens. Rev. Bryol. Lichenol. 32: 166-180.

Grolle, R. (1972) Miscellanea hepaticologica 121-130. J. Hattori Bot. Lab. 36: 547-551.

Grolle, R. (1979) Miscellanea hepaticologica 171-180. J. Bryol. 10(3): 163-72.

Hatcher, R.E. (1960) A monograph of the genus Isotachis. Nova Hedwigia 2(4): 573-608.

Hattori, S. (1979a) A revision of the Australasian species of the genus Frullania, Hepaticae, I. J. Hattori Bot. Lab. 45: 323-63.

Hattori, S. (1979b) A revision of the Australasian species of the genus Frullania, Hepaticae, II. J. Hattori Bot. Lab. 46: 119-53.

Hattori, S. (1983) A revision of Australasian species of Frullania, Hepaticae. III. J. Hattori Bot. Lab. 54: 133-182.

Hattori, S. (1985) Two Australian species of the genus Porella L. (Hepaticae). Bull. Natl Sci. Mus., Tokyo, Ser. B., 11(2): 51-55. 
Hattori, S. (1987) New or little known species of Frullania (Frullaniaceae) from Queensland and New South Wales, Australia. Mem. New York Bot. Gard. 45: 544-555.

Hewson, H.J. (1970a) The family Aneuraceae in Australia and New Guinea: I. The genus Aneura. Proc. Linn. Soc. New South Wales 94(2): 173-193.

Hewson, H.J. (1970b) The family Aneuraceae in Australia and New Guinea: II. The genus Riccardia. Proc. Linn. Soc. New South Wales 95(1): 60-121.

Hicks, M.L. (1992) Queensland liverworts. Reboulia Raddi. J. Hattori Bot. Lab. 71: 113-117.

Inoue, H. (1986) Notes on the Plagiochilaceae XIII. Review of the genus Plagiochila (Dum.) Dum. in mainland Australia. J. Hattori Bot. Lab. 60: 357-378.

Inoue, H. \& Schuster, R.M. (1971) A monograph of the New Zealand and Tasmanian Plagiochilaceae. J. Hattori Bot. Lab. 34: 1-225.

Na-Thalang, O. (1980) A revision of the genus Riccia (Hepaticae) in Australia. Brunonia 3: 61-140.

Ramsay, H.P. (1980) Contributions of Rev. W.W. Watts F.L.S. to Australian botany. Taxon 29: 455-469.

Ramsay, H.P. \& Seur, J. (1990) Type specimens of bryophytes in Australian herbaria. Pp. 247252 in P.S. Short (ed.), History of systematic botany in Australasia. (Australian Systematic Botany Society Inc: Collingwood)

Ramsay, H.P., Seur, J., Wilson, P.G. \& Goodwin, T. (1990) Register of type specimens of mosses in Australian Herbaria. General introduction and part 1. Special collections at NSW: Lord Howe Island, Vanuatu (New Hebrides). Telopea 3: 571-592.

Rodway, L. (1918) Tasmanian bryophyta. Pap. Proc. Roy. Soc. Tasmania 1916: 51-143.

Schuster, R.M. (1960) Studies on Hepaticae. II. The new family Chaetophyllopsidaceae. J. Hattori Bot. Lab. 23: 68-76.

Schuster, R.M. (1980) Studies on Hepaticae, LIV-LVIII. Kurzia v. Mart. [Microledipodzia (Spr.) Joerg.l, Mitt., Drucella Hodgs. and Isolembidium Schust. J. Hattori Bot. Lab. 48: 337-421.

Scott, G.A.M. (1985) Southern Australian Liverworts. Australian Flora and Fauna Series 2.

Scott, G.A.M. \& Bradshaw, J.A. (1986) Australian liverworts (Hepaticae): annotated list of binomials and check-list of published species with bibliography. Brunonia 8: 1-171.

Scott, G.A.M. \& Pike, D.C. (1988) Revisionary notes on Fossombronia. Bryologist 91(3): 193-201.

Stephani, F. (1884) Die Gattung Radula. Hedwigia 23: 113-6, 129-137, 145-9, 161-3.

Stephani, F. (1889) Hepaticae Australiae 1-3. Hedwigia 28: 128-135, 155-175, 257-278.

Stephani, F. (1906-09) Species Hepaticarum 3 (Georg: Geneva) [Relevant page dates: 517-693 (1909)]

Stephani, F. (1909-12) Species Hepaticarum 4 (Georg: Geneva) [Relevant page dates: 97-448 (1910); 449-752 (1911); 753-824 (1912)]

Stephani, F. (1912-17) Species Hepaticarum 5 (Georg: Geneva) [Relevant page dates: 1-176 (1912); 849-1008 (1916)]

Stephani, F. (1917-25) Species Hepaticarum 6 (Georg: Geneva) [Relevant page dates 1-128 (1917); 129-176 (1918); 177-240 (1921)]

Stephani, F. \& Watts, W.W. (1914) Hepaticae Australes. J. \& Proc. Roy. Soc. New South Wales 48: 94-135.

Thiers, B.M. (1988) The Australian species of Cololejeunea. Beiheft Nova Hedwigia 90: 113-146.

Thiers, B.M. \& Gradstein, R. (1989) Lejeuneaceae (Hepaticae) of Australia. I. Subfamily Ptychanthoideae. Mem. New York Bot. Garden 52: 1-82.

Watts, W.W. (1902) Notes on some Richmond River hepatics. Proc. Linn. Soc. New South Wales 26: $215-216$.

Watts, W.W. (1903) Notes on some Australian hepatics. Proc. Linn. Soc. New South Wales 27: 493494.

Watts, W.W. (1905) Further notes on Australian hepatics. Proc. Linn. Soc. New South Wales 29: $558-560$.

Watts, W.W. (1912) Census of New South Wales hepatics. Royal Botanic Gardens, Sydney. (Unpublished manuscript)

Watts, W.W. \& Whitelegge, T. (1902) Census Muscorum Australiensium. Proc. Linn. Soc. New South Wales suppl. 27: 1-90.

Watts, W.W. \& Whitelegge, T. (1906) Census Muscorum Australiensium. Proc. Linn. Soc. New South Wales suppl. 30: 91-163. 